\title{
Estimation of Open Boundary Conditions Based on an Isopycnic-Coordinate Internal Tidal Model with Adjoint Assimilation Method
}

\author{
Yanqiu Gao, ${ }^{1}$ Guangzhen Jin, ${ }^{1}$ Haibo Chen, ${ }^{1,2}$ and Xianqing Lv $^{1}$ \\ ${ }^{1}$ Laboratory of Physical Oceanography, Ocean University of China, Qingdao 266100, China \\ ${ }^{2}$ China Offshore Environmental Services Ltd., Qingdao 266061, China \\ Correspondence should be addressed to Xianqing Lv; xqinglv@ouc.edu.cn
}

Received 19 December 2012; Revised 14 May 2013; Accepted 27 May 2013

Academic Editor: Yuping Wang

Copyright (C) 2013 Yanqiu Gao et al. This is an open access article distributed under the Creative Commons Attribution License, which permits unrestricted use, distribution, and reproduction in any medium, provided the original work is properly cited.

The isopycnic-coordinate internal tidal model with adjoint assimilation method is developed into a three-layer version. Two groups of ideal experiments are carried out in order to investigate the estimation of spatially varying open boundary conditions (OBCs). In group 1, different independent point schemes (IPSs) are used to invert 6 kinds of prescribed distributions of OBCs. Results show that, after assimilation, the cost functions and their gradient norms are reduced by about 2 orders of magnitude and by about 1 order of magnitude, respectively; the mean absolute errors (MAEs) in OBCs and the vector differences of horizontal current are reduced by 1 order of magnitude and by more than $23.53 \%$ compared with the values before assimilation, respectively. The results demonstrate that the three-layered model has a good ability in estimating the spatially varying OBCs; the use of IPSs can effectively improve the estimation precision; fewer independent points are feasible when the distribution is simpler, and appropriately more independent points are required when the distribution is more complex. In group 2, by using the optimal IPSs in group 1, the model is also able to successfully invert the OBCs on a real topography in the Luzon Strait area. The results are important to the study of the internal tide in the South China Sea.

\section{Introduction}

Internal tides play an important role in the dissipation of barotropic tides and in mixing the deep ocean $[1,2]$ and consequently contribute to the large-scale ocean circulation. Numerical simulation is an effective mean to study internal tides $[3,4]$. The open boundary conditions (OBCs) are very important in regional ocean modeling and have a critical impact on the simulation results of the internal tides. The treatment of $\mathrm{OBCs}$ has been investigated by many researchers [5-10].

The adjoint assimilation method has been widely used for parameter estimation. Its basic idea is as follows. First, a forward model is defined by a series of control equations and several initial and boundary conditions. Second, an adjoint model is derived based on the forward model. The cost function keeps decreasing while the parameters are optimized by the adjoint model, achieving an organic combination of numerical model and observations. By assimilating the data in the interior region, the adjoint assimilation method can optimize the parameters automatically and reach a global optimization. In a 3D shallow water flow system (TRIWAQ), Heemink et al. [11] used the adjoint approach to estimate the harmonic constants at the open boundaries, the friction parameter, the viscosity parameter, and the water depth by assimilating the tide gauge data as well as the altimeter data. Taillandier et al. [12] developed a four-dimensional variational data-assimilation approach to estimate the initial and open boundary conditions that force a coastal model according to interior observations. Ayoub [13] carried out experiments to estimate the OBCs in a North Atlantic circulation model using the adjoint method. Gejadze and Copeland [14] studied the open boundary control problem for freesurface barotropic Navier-Stokes equations with adjoint data assimilation technology. Zhang and $\mathrm{Lu}$ [15] optimized the OBCs and simulated the $M_{2}$ tide and tidal current in the Bohai and Yellow Seas by assimilating satellite altimetry. Kazantsev [16] used a variational data assimilation technique 
to control boundary conditions on rigid boundaries for a shallow-water model. Chen et al. [17] constructed an adjoint assimilation model for simulation of internal tides and simply tested with a series of ideal experiments in which several prescribed spatial distributions of OBCs were successfully inverted by assimilating the model-generated pseudoobservations. Cao et al. [18] and Guo et al. [19] studied the inversion of OBCs by assimilating the $T / P$ altimeter data with adjoint assimilation method in a $2 \mathrm{D}$ tidal model. Cao et al. [20] optimized OBCs in a 3D internal tidal model with the adjoint method around Hawaii.

In this paper, the isopycnic-coordinate internal tidal model constructed by Chen et al. [17] is developed into a three-layer version in order to investigate the estimation of spatially varying OBCs. Two groups of ideal experiments are carried out. One group is used to verify the efficiency of the model on an ideal topography and to test the ability of the model in estimation of the spatially varying OBCs. In the other group, we estimate the OBCs on a real topography in the Luzon Strait area using the optimal independent point schemes (IPSs) obtained in the former group. This paper is organized as follows. The isopycnic-coordinate internal tidal model with adjoint assimilation method, the optimization method, and the experimental design are briefly introduced in Section 2. The experimental results are discussed in detail in Section 3. Finally, we make a summary and draw some conclusions in Section 4.

\section{Model and Experimental Design}

The isopycnic-coordinate numerical model for simulation of internal tides with adjoint assimilation method is composed of a forward model and its adjoint model. The forward model is used to simulate the internal tides and the adjoint model is used to optimize the modal control variables. Based on this assimilation model, two groups of ideal experiments are carried out to investigate the estimation of spatially varying OBCs.

2.1. Isopycnic-Coordinate Internal Tidal Model with Adjoint Assimilation Method. The isopycnic-coordinate internal tidal model employs spherical coordinates in horizontal direction and isopycnic coordinates in the vertical one. This model is based on the nonlinear, time-dependent, and free-surface hydrostatic equations, and is subject to the hydrostatic approximations. The internal mode equations are as follows: upper layer:

$$
\begin{gathered}
\frac{\partial q_{1}}{\partial t}+\frac{1}{R \cos \varphi} \frac{\partial\left(q_{1} u_{1}\right)}{\partial \lambda}+\frac{1}{R \cos \varphi} \frac{\partial\left(q_{1} v_{1} \cos \varphi\right)}{\partial \varphi}=0 \\
\frac{\partial u_{1}}{\partial t}+\frac{u_{1}}{R \cos \varphi} \frac{\partial u_{1}}{\partial \lambda}+\frac{v_{1}}{R} \frac{\partial u_{1}}{\partial \varphi}-\frac{u_{1} v_{1} \tan \varphi}{R}-f v_{1} \\
+\frac{g}{R \cos \varphi} \frac{\partial}{\partial \lambda} \sum_{m=1}^{3}\left(\frac{q_{m}}{\rho_{m}}-h_{m}\right) \\
-A_{h 1} \Delta u_{1}+\frac{A_{v 1} \bar{\rho}_{1}}{q_{1} \bar{h}_{1}}\left(u_{1}-u_{2}\right)=0
\end{gathered}
$$

$$
\begin{aligned}
\frac{\partial v_{1}}{\partial t}+ & \frac{u_{1}}{R \cos \varphi} \frac{\partial v_{1}}{\partial \lambda}+\frac{v_{1}}{R} \frac{\partial v_{1}}{\partial \varphi}+\frac{u_{1}^{2} \tan \varphi}{R} \\
& +f u_{1}+\frac{g}{R} \frac{\partial}{\partial \varphi} \sum_{m=1}^{3}\left(\frac{q_{m}}{\rho_{m}}-h_{m}\right) \\
& -A_{h 1} \Delta v_{1}+\frac{A_{v 1} \bar{\rho}_{1}}{q_{1} \bar{h}_{1}}\left(v_{1}-v_{2}\right)=0,
\end{aligned}
$$

the second layer:

$$
\begin{gathered}
\frac{\partial q_{2}}{\partial t}+\frac{1}{R \cos \varphi} \frac{\partial\left(q_{2} u_{2}\right)}{\partial \lambda}+\frac{1}{R \cos \varphi} \frac{\partial\left(q_{2} v_{2} \cos \varphi\right)}{\partial \varphi}=0 \\
\frac{\partial u_{2}}{\partial t}+\frac{u_{2}}{R \cos \varphi} \frac{\partial u_{2}}{\partial \lambda}+\frac{v_{2}}{R} \frac{\partial u_{2}}{\partial \varphi}-\frac{u_{2} v_{2} \tan \varphi}{R}-f v_{2} \\
+\frac{g}{R \cos \varphi} \frac{\partial}{\partial \lambda} \sum_{m=1}^{3}\left(\frac{q_{m}}{\rho_{2}}-h_{m}\right)-A_{h 2} \Delta u_{2} \\
-\frac{A_{v 1} \bar{\rho}_{1}}{q_{2} \bar{h}_{1}}\left(u_{1}-u_{2}\right)+\frac{A_{v 2} \bar{\rho}_{2}}{q_{2} \bar{h}_{2}}\left(u_{2}-u_{3}\right) \\
+\frac{g}{R \cos \varphi} \sum_{m=1}^{2}\left(\frac{1}{\rho_{2}}-\frac{1}{\rho_{m}}\right) \frac{\partial q_{m}}{\partial \varphi}=0 \\
\frac{\partial v_{2}}{\partial t}+\frac{u_{2}}{R \cos \varphi} \frac{\partial v_{2}}{\partial \lambda}+\frac{v_{2}}{R} \frac{\partial v_{2}}{\partial \varphi}+\frac{u_{2}^{2} \tan \varphi}{R}+f u_{2} \\
+\frac{g}{R} \frac{\partial}{\partial \varphi} \sum_{k=1}^{3}\left(\frac{q_{k}}{\rho_{2}}-h_{k}\right)-A_{h 2} \Delta v_{2}-\frac{A_{v 1} \bar{\rho}_{1}}{q_{2} \bar{h}_{1}}\left(v_{1}-v_{2}\right) \\
\left.q_{2}-v_{3}\right)+\frac{g}{R} \sum_{m=1}^{2}\left(\frac{1}{\rho_{2}}-\frac{1}{\rho_{m}}\right) \frac{\partial q_{m}}{\partial \varphi}=0
\end{gathered}
$$

lower layer:

$$
\begin{gathered}
\frac{\partial q_{3}}{\partial t}+\frac{1}{R \cos \varphi} \frac{\partial\left(q_{3} u_{3}\right)}{\partial \lambda}+\frac{1}{R \cos \varphi} \frac{\partial\left(q_{3} v_{3} \cos \varphi\right)}{\partial \varphi}=0 \\
\frac{\partial u_{3}}{\partial t}+\frac{u_{3}}{R \cos \varphi} \frac{\partial u_{3}}{\partial \lambda}+\frac{v_{3}}{R} \frac{\partial u_{3}}{\partial \varphi}-\frac{u_{3} v_{3} \tan \varphi}{R}-f v_{3} \\
+\frac{g}{R \cos \varphi} \frac{\partial}{\partial \lambda} \sum_{m=1}^{3}\left(\frac{q_{m}}{\rho_{3}}-h_{m}\right)-A_{h 3} \Delta u_{3} \\
-\frac{A_{v 2} \bar{\rho}_{2}}{q_{3} \bar{h}_{2}}\left(u_{2}-u_{3}\right) \\
+\frac{\kappa \rho_{3} u_{3}}{q_{3}} \sqrt{\left(u_{3}\right)^{2}+\left(v_{3}\right)^{2}}=0
\end{gathered}
$$




$$
\begin{aligned}
\frac{\partial v_{3}}{\partial t}+ & \frac{u_{3}}{R \cos \varphi} \frac{\partial v_{3}}{\partial \lambda}+\frac{v_{3}}{R} \frac{\partial v_{3}}{\partial \varphi}+\frac{u_{3}^{2} \tan \varphi}{R}+f u_{3} \\
& +\frac{g}{R} \frac{\partial}{\partial \varphi} \sum_{m=1}^{3}\left(\frac{q_{m}}{\rho_{3}}-h_{m}\right)-A_{h 3} \Delta v_{3}-\frac{A_{v_{2}} \bar{\rho}_{2}}{q_{3} \bar{h}_{2}}\left(v_{2}-v_{3}\right) \\
& +\frac{\kappa \rho_{3} v_{3}}{q_{3}} \sqrt{\left(u_{3}\right)^{2}+\left(v_{3}\right)^{2}}=0 .
\end{aligned}
$$

Here, $t$ is the time, $\lambda$ and $\varphi$ are the east longitude and north latitude, respectively, $u_{m}(\lambda, \varphi, t)$ and $v_{m}(\lambda, \varphi, t)$ are horizontal velocities in $\lambda$ and $\varphi$ in the $m$ th $(m=1,2,3$, surface for $m=1)$ layer, respectively, and $q_{m}(\lambda, \varphi, t)$ is the timevarying layer mass and

$$
\begin{gathered}
q_{1}=\rho_{1}\left(h_{1}+\eta_{1}-\eta_{2}\right), \\
q_{2}=\rho_{2}\left(h_{2}+\eta_{2}-\eta_{3}\right), \\
q_{3}=\rho_{3}\left(h_{3}+\eta_{3}\right),
\end{gathered}
$$

where $h_{m}$ and $\eta_{m}$ are the undisturbed layer thickness and interface elevation above the undisturbed level, respectively. $R$ is the radius of the earth, $g$ is the gravitational acceleration, $f$ is the Coriolis parameter, $A_{h m}$ is the horizontal eddy viscosity coefficient in the $m$ th layer, respectively, $\Delta$ is the Laplace operator, and

$$
\Delta(u, v)=\frac{1}{R^{2} \cos ^{2} \varphi} \frac{\partial^{2}(u, v)}{\partial \lambda^{2}}+\frac{1}{R^{2} \cos \varphi} \frac{\partial}{\partial \varphi}\left[\cos \varphi \frac{\partial(u, v)}{\partial \varphi}\right]
$$

$A_{v m}(m=1,2)$ and $\kappa$ are the interface and bottom friction coefficients, respectively. $\bar{\rho}_{m}=\left(\rho_{m}+\rho_{m+1}\right) / 2$ and $\bar{h}_{m}=\left(h_{m}+\right.$ $\left.h_{m+1}\right) / 2(m=1,2)$, where $\rho_{m}$ is the potential density which is constant in each layer. In the forward model, $q, u$, and $v$ are the main output and called the state variables in this paper.

The barotropic currents are defined by

$$
U=\frac{\left(q_{1} u_{1}+q_{2} u_{2}+q_{3} u_{3}\right)}{Q}, \quad V=\frac{\left(q_{1} v_{1}+q_{2} v_{2}+q_{3} v_{3}\right)}{Q},
$$$$
\text { where } Q=q_{1}+q_{2}+q_{3} \text {. }
$$

Integrating the continuity equations and the momentum equations in the vertical, we get the external mode as follows:

$$
\begin{aligned}
& \frac{\partial Q}{\partial t}+\frac{1}{R \cos \varphi} \frac{\partial(Q U)}{\partial \lambda}+\frac{1}{R \cos \varphi} \frac{\partial(Q V \cos \varphi)}{\partial \varphi}=0, \\
& \frac{\partial U}{\partial t}-f V+M_{\lambda}+R_{U}+\frac{\kappa \rho_{3} u_{3}}{Q} \sqrt{\left(u_{3}\right)^{2}+\left(v_{3}\right)^{2}}=0, \\
& \frac{\partial V}{\partial t}+f U+M_{\varphi}+R_{V}+\frac{\kappa \rho_{3} v_{3}}{Q} \sqrt{\left(u_{3}\right)^{2}+\left(v_{3}\right)^{2}}=0,
\end{aligned}
$$

where

$$
\begin{aligned}
M_{\lambda}= & \frac{g}{R \cos \varphi} \frac{\partial}{\partial \lambda}\left(\frac{Q}{\rho}-h\right) \\
& +\frac{g}{Q R \cos \varphi} \sum_{k=2}^{3}\left[q_{k} \sum_{m=1}^{2}\left(\frac{1}{\rho_{k}}-\frac{1}{\rho_{m}}\right) \frac{\partial q_{m}}{\partial \lambda}\right], \\
M_{\varphi}= & \frac{g}{R} \frac{\partial}{\partial \varphi}\left(\frac{Q}{\rho}-h\right)+\frac{g}{Q R} \sum_{k=2}^{3}\left[q_{k} \sum_{m=1}^{2}\left(\frac{1}{\rho_{k}}-\frac{1}{\rho_{m}}\right) \frac{\partial q_{m}}{\partial \varphi}\right], \\
R_{U}= & \frac{1}{Q} \sum_{m=1}^{3} q_{m}\left(\frac{u_{m}}{R \cos \varphi} \frac{\partial u_{m}}{\partial \lambda}+\frac{v_{m}}{R} \frac{\partial u_{m}}{\partial \varphi}-\frac{u_{m} v_{m} \tan \varphi}{R}\right) \\
& -\frac{1}{Q} \sum_{m=1}^{3} q_{m} A_{h m} \Delta u_{m}-\sum_{m=1}^{3} u_{m} \frac{\partial}{\partial t}\left(\frac{q_{m}}{Q}\right), \\
R_{V}= & \frac{1}{Q} \sum_{m=1}^{3} q_{m}\left(\frac{u_{m}}{R \cos \varphi} \frac{\partial v_{m}}{\partial \lambda}+\frac{v_{m}}{R} \frac{\partial v_{m}}{\partial \varphi}+\frac{u_{m}^{2} \tan \varphi}{R}\right) \\
& -\frac{1}{Q} \sum_{m=1}^{3} q_{m} A_{h m} \Delta v_{m}-\sum_{m=1}^{3} v_{m} \frac{\partial}{\partial t}\left(\frac{q_{m}}{Q}\right) .
\end{aligned}
$$

Here $h(\lambda, \varphi)\left(=h_{1}+h_{2}+h_{3}\right)$ is the undisturbed water depth; $\rho(\lambda, \varphi, t)$ is the vertically averaged density and $\rho=$ $Q /\left(h+\eta_{1}\right)$. Note that the second terms of $M_{\lambda}$ and $M_{\varphi}$ represent the resultant effect of the horizontal density gradients in all layers on the sea surface and are the main causes of the surface manifestation of internal tides.

Based on the governing equations (1)-(2.1) of the forward model, its adjoint model can be constructed. Similar to the forward model, the adjoint model also consists of the internal and external modes. The details of the adjoint model in a generalized form can be found in the work of Chen et al. [17].

The cost function measuring the data misfit between the model output and observations is minimized through optimizing the control parameters. The cost function is defined as

$$
\begin{aligned}
& \mathbf{J}(q, u, v ; \mathbf{p}) \\
& \quad=\frac{1}{2} \int_{\Sigma}\left[K_{u} \sum_{m=1}^{3}\left(u_{m}-\widehat{u}_{m}\right)^{2}+K_{v} \sum_{m=1}^{3}\left(v_{m}-\widehat{v}_{m}\right)^{2}\right] d \sigma,
\end{aligned}
$$

where $m(m=1,2,3)$ is layer index, $\sum$ denotes the computing area of both time and space, $\mathbf{p}$ represents the generalized control parameters, $u_{m}$ and $v_{m}$ are the model simulated, and $\widehat{u}_{m}$ and $\widehat{v}_{m}$ are the observations. Here $K_{u}$ and $K_{v}$ are the weight matrices and theoretically should be the inverse of the observation error covariance matrix. In this paper, since only ideal experiments with model-generated pseudoobservations are performed, by assuming that the errors in the data are uncorrelated and equally weighted, and $K_{u}$ and $K_{v}$ are reduced to unit matrices [21]. 
The Lagrangian function is defined by

$$
\begin{aligned}
& \mathbf{L}\left(q, u, v ; q_{a}, u_{a}, v_{a} ; \mathbf{p}\right) \\
& =\mathbf{J}(q, u, v ; \mathbf{p}) \\
& \quad+\sum_{m=1}^{3} \int_{\Sigma}\left[q_{a m} \cdot \text { Eq. }(3 m-2)+u_{a m} q_{m} \cdot \text { Eq. }(3 m-1)\right. \\
& \left.+v_{a m} q_{m} \cdot \text { Eq. }(3 m)\right] d \sigma,
\end{aligned}
$$

where $q_{a m}, u_{a m}$, and $v_{a m}$ are called adjoint variables of state variables $q_{m}, u_{m}$, and $v_{m}$, respectively.

According to the typical theory of Lagrangian multiplier method, we have the following first-order derivates of Lagrangian function with respect to all the variables and parameters:

$$
\begin{array}{rlrl}
\frac{\partial \mathbf{L}}{\partial q_{a m}}=0, & \frac{\partial \mathbf{L}}{\partial u_{a m}}=0, & & \frac{\partial \mathbf{L}}{\partial v_{a m}}=0 ; \\
\frac{\partial \mathbf{L}}{\partial q_{m}}=0, & \frac{\partial \mathbf{L}}{\partial u_{m}}=0, & \frac{\partial \mathbf{L}}{\partial v_{m}}=0 ; \\
\frac{\partial \mathbf{L}}{\partial \mathbf{p}}=0 . &
\end{array}
$$

Equation (19) returns the governing equations (1)-(2.1). The adjoint equations can be derived from (20). From (21) we can obtain the gradients of the cost function with respect to control parameters.

In the model, OBC at the $n$th time step along the open boundary at a certain open boundary grid point $(I, J)$ is described as

$$
\eta_{I, J}^{n}=a_{I, J} \cos (\omega n \Delta t)+b_{I, J} \sin (\omega n \Delta t)
$$

where $\eta$ is water level, $\omega$ denotes the frequency of $M_{2}$ constituent, $\Delta t$ is the time step length, and $a_{I, J}$ and $b_{I, J}$ are the Fourier coefficients as well as the tunable parameters of the model. The gradients of cost function with respect to $a_{I, J}$ and $b_{I, J}$ can be deduced from (21) which yields

$$
\begin{gathered}
\frac{\partial \mathbf{J}}{\partial a_{I, J}}+\sum_{n} T_{I, J}^{n} \cos (\omega n \Delta t)=0, \\
\frac{\partial \mathbf{J}}{\partial b_{I, J}}+\sum_{n} T_{I, J}^{n} \sin (\omega n \Delta t)=0,
\end{gathered}
$$

where $T_{I, J}^{n}$ refers to Chen et al. [17].

So the inverse problem about the OBCs is transformed into the one about Fourier coefficients $a$ and $b$.

2.2. Experimental Design. Based on the three-layered assimilation model, two groups of ideal experiments (i.e., groups 1 and 2) are carried out to investigate the influence of IPS on the OBCs estimation. In order to test the ability in estimating the spatially varying OBCs in a nutshell, only $a$ (one of Fourier coefficients) along east boundary is estimated in this paper.

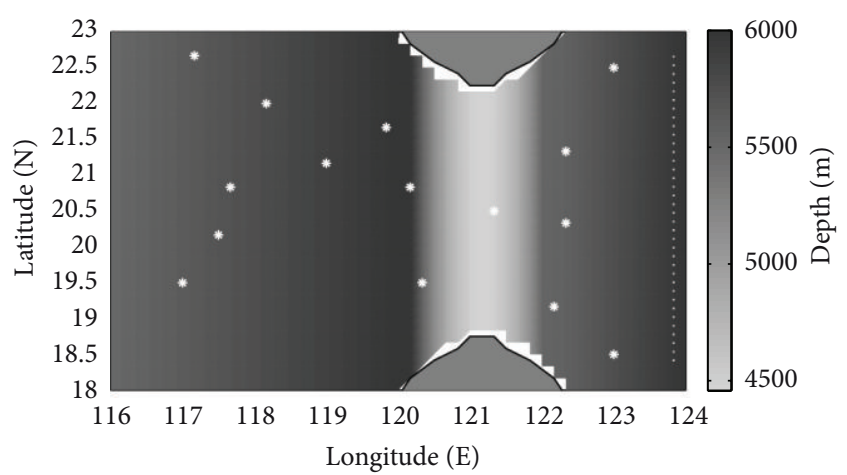

FIGURE 1: Plan view of calculation region of group 1.

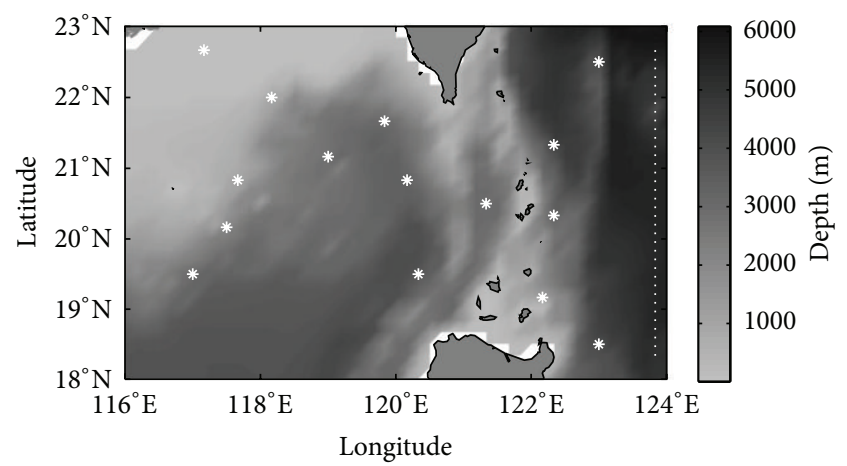

Figure 2: Plan view of calculation region of group 2.

Meanwhile, other open boundary conditions are set to be known.

In group 1, on an ideal topography, six kinds of spatial distributions of OBCs are inverted with this three-layered model. Different IPSs are employed in accordance with the spatial distributions of OBCs. The optimal schemes are selected after comparing the results of group 1 and are used to estimate the OBCs in group 2 in which a real topography in the Luzon Strait is employed.

The calculation regions (from $116^{\circ} \mathrm{E}$ to $124^{\circ} \mathrm{E}$ and from $18^{\circ} \mathrm{N}$ to $23^{\circ} \mathrm{N}$ ) of groups 1 and 2 are shown in Figures 1 and 2 , respectively. In both figures, the white stars denote the 15 observation locations and the white dots denote the 27 open boundary points where the barotropic tidal force is installed. The horizontal resolution in this model is $10^{\prime} \times 10^{\prime}$, and there are $49 \times 31$ grids totally in this area. The two undisturbed interfaces of this three-layered model are placed at the depth of $200 \mathrm{~m}$ and $400 \mathrm{~m}$, respectively, and the potential densities in three layers are $1023.42 \mathrm{~kg} / \mathrm{m}^{3}, 1025.02 \mathrm{~kg} / \mathrm{m}^{3}$, and $1026.77 \mathrm{~kg} / \mathrm{m}^{3}$, respectively, based on the temperaturesalinity data of the world oceans data sets (WOA05). The Coriolis parameter is set to be local value, the angular frequency of $M_{2}$ tide is $1.4050789025 \times 10^{-4} \mathrm{~s}^{-1}$, and the whole-time step is $496.863 \mathrm{~s}$ ( $1 / 90$ of the period of $M_{2}$ tide) for both external and internal modes. The time of simulation is equal to 20 periods of $M_{2}$ tide. The horizontal eddy viscosity coefficient is chosen to be $A_{h}=1000$. The coefficients of bottom and interface friction are taken as $\kappa=0.003$ 

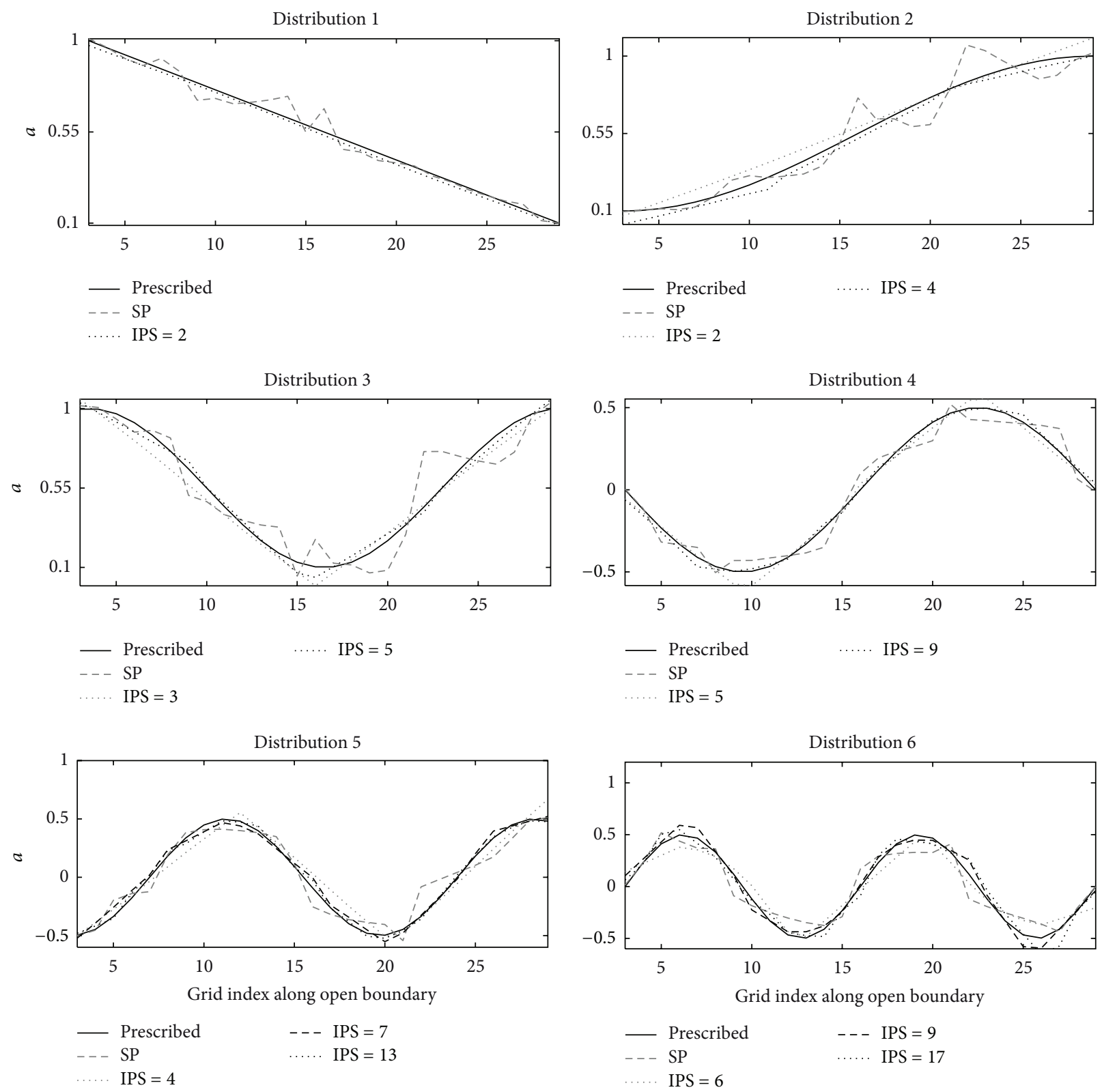

FIGURE 3: Six kinds of prescribed OBCs (the black solid lines) and their inverted distributions.

and $A_{v}=0.005$, respectively. Every observation location has "observations" of tidal current, if possible, in the upper two layers. The Flather conditions are installed along four boundaries and, without loss of generality, the unknown open boundary is supposed to be east boundary.

The 6 kinds of prescribed distributions of OBCs in group 1 are shown in Figure 3. First of all, direct inversion without IPS for each kind of distribution is carried out as a comparison experiment. Next, different number of independent points (Table 1) is employed according to the complexity of $\mathrm{OBC}$ distributions. The $\mathrm{OBCs}$ at each point along the east boundary can be derived from linear interpolation (the Cressman interpolation is employed in this paper) of values at independent points.
TABLE 1: The distributions of OBCs and the IPSs.

\begin{tabular}{lccc}
\hline Distributions & \multicolumn{3}{c}{ The number of independent points } \\
\hline 1 & 2 & - & - \\
2 & 2 & 4 & - \\
3 & 3 & 5 & - \\
4 & 5 & 9 & - \\
5 & 4 & 7 & 13 \\
6 & 6 & 9 & 17 \\
\hline
\end{tabular}

For each distribution there is an optimal IPS in group 1. The optimal IPSs are selected to invert the 6 kinds of prescribed distributions of OBCs in group 2. 
TABLE 2: The MAEs in OBCs before assimilation (BA) and after assimilation (AA). The values in parentheses are the optimal numbers of independent points.

\begin{tabular}{lccccrr}
\hline Distributions & $1(2)$ & $2(4)$ & $3(5)$ & $4(9)$ & $5(13)$ & $6(17)$ \\
\hline BA & 0.550 & 0.550 & 0.567 & 0.305 & 0.326 & 0.305 \\
AA & 0.017 & 0.033 & 0.029 & 0.020 & 0.023 & 0.058 \\
\hline
\end{tabular}

TABLE 3: Statistics of the errors before assimilation (BA) and after assimilation (AA). The values in parentheses in the first column are the optimal numbers of independent points and the unit of the errors is $\mathrm{cms}^{-1}$.

\begin{tabular}{|c|c|c|c|c|c|c|}
\hline \multirow{2}{*}{ Distributions } & \multicolumn{3}{|c|}{$u$-vector difference } & \multicolumn{3}{|c|}{$v$-vector difference } \\
\hline & BA & $\mathrm{AA}$ & 1-AA/BA (\%) & BA & $\mathrm{AA}$ & 1-AA/BA (\%) \\
\hline $1(2)$ & 2.65 & 0.19 & 92.83 & 0.92 & 0.17 & 81.52 \\
\hline $2(4)$ & 2.72 & 0.40 & 85.29 & 0.85 & 0.60 & 29.41 \\
\hline $3(5)$ & 1.45 & 0.07 & 95.17 & 0.32 & 0.12 & 62.50 \\
\hline $4(9)$ & 0.43 & 0.19 & 55.81 & 0.41 & 0.11 & 83.17 \\
\hline $5(13)$ & 0.37 & 0.05 & 86.49 & 0.18 & 0.06 & 66.67 \\
\hline $6(17)$ & 0.27 & 0.20 & 25.93 & 0.17 & 0.13 & 23.53 \\
\hline
\end{tabular}

\section{Experimental Results and Discussions}

The forward model is run with certain prescribed OBCs, and the model-generated results of the currents (i.e., currents in the upper two layers) at these observation locations are taken as the pseudo-observations. Then, an initial value (taken as zero here) of $a$ is assigned to run the forward model. The difference between the simulated values and pseudoobservations plays the role of the external force of the adjoint model. The optimized OBCs can be obtained through the backward integration of the adjoint equations. The inverse integral time of the adjoint equations is equal to a period of $M_{2}$ tide. With the procedure above repeated, the OBCs will be optimized continually and the difference between simulated values and pseudo-observations will be diminished. Meanwhile, the difference between the prescribed and the estimated OBCs is also decreased. Different iteration numbers are tried in group 1, and 100 iterations are good choice based on the following considerations. (1) The misfit between "observations" and the simulated results is very small and approximately constant when it declines to a certain value after 100 iterations; (2) the cost functions are almost no longer falling after 100 iterations; (3) the calculation amount is acceptable for the 100 iterations. In group 2, the iteration number is also taken as 100 .

3.1. Results and Discussions of Group 1. In this section, six kinds of spatial distributions of OBCs are inverted on an ideal topography. The results are shown in Figure 3. The prescribed distributions are all inverted successfully. The inverted distributions obtained without IPS have some obvious fluctuations, while those obtained with IPSs show good consistency with the prescribed distributions. The results demonstrate that the model has a good ability in estimating the spatially varying
OBCs. Moreover, the IPSs can further improve the estimation precision.

The mean absolute errors (MAEs) between the prescribed OBCs and the values show that utilization of fewer independent points is feasible when the distribution of OBCs is simpler, and appropriately more independent points can lead to higher precision when the distribution of OBCs is more complex. The result that the use of IPSs can indeed effectively improve the precision of parameter estimation is consistent with the results in [22-24]. The MAEs between the prescribed OBCs and the values obtained with the optimal IPSs corresponding to the 6 kinds of spatial distributions are shown in Table 2. The MAEs in OBCs are reduced by 1 order of magnitude compared with the values before assimilation. The results indicate that the model performs great effect on estimating the OBCs with the optimal IPSs.

The cost function and the $L_{2}$ norms of its gradient with respect to OBCs are two important criteria to evaluate the assimilation efficiency. The iteration histories of the cost functions and their gradient norms (all values are normalized by their own initial values) for the 6 kinds of distributions of OBCs are shown in Figures 4 and 5 (only the histories of the previous 50 iterations are plotted), respectively.

The cost functions are declining dramatically during the assimilation process. After 100 iterations, the cost functions and their gradient norms are reduced by about 2 orders of magnitude and by about 1 order of magnitude compared with their initial values, respectively. The declining cost functions and gradient norms indicate that the OBCs have been indeed optimized and are getting close to their prescribed values.

Statistics of the vector differences in horizontal tidal current before and after assimilation are shown in Table 3. From Table 3 we can see that the vector differences in horizontal tidal current are reduced by more than $23.53 \%$ 

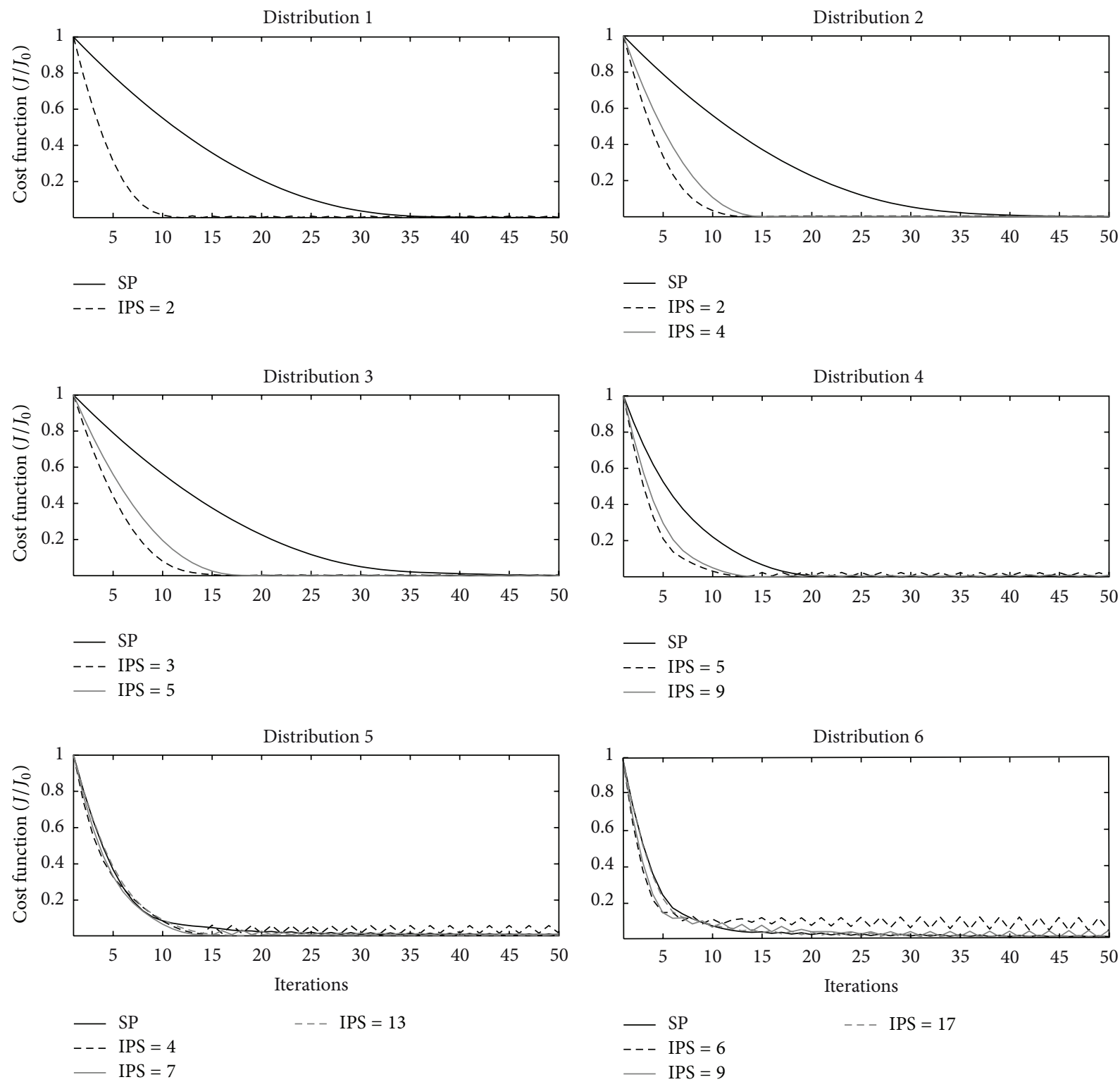

FIGURE 4: The iteration histories of the cost functions for group 1.

compared with the values before assimilation. It indicates that all the 6 kinds of spatial distributions of OBCs have been inverted successfully.

Different IPSs are employed to invert the 6 kinds of prescribed distributions of OBCs in group 1. After 100 iterations, the cost functions and their gradient norms are reduced by about 2 orders of magnitude, and by about 1 order of magnitude, respectively, compared with their initial values. Meanwhile, the MAEs in OBCs are reduced by about 1 order of magnitude, and the vector differences in horizontal tidal current are reduced by more than $23.53 \%$ compared with their values before assimilation. The results demonstrate that the model is effective and it has a good ability in estimating the spatially varying OBCs. Moreover, utilization of fewer independent points is feasible when the distribution of OBCs is simpler, and appropriately more independent points are required when the distribution of OBCs to be inverted is more complex. For each prescribed distribution of OBCs, the IPSs leading to the best result is taken as the optimal one. According to the results of group 1, the optimal numbers of independent points are $2,4,5,9,13$, and 17 corresponding to the 6 kinds of prescribed OBC distributions, respectively.

3.2. Results and Discussions of Group 2. In this section, six experiments are carried out on a real topography in the Luzon Strait area. Corresponding to these 6 experiments, six kinds of prescribed distributions of OBCs are inverted, respectively, with the optimal IPSs derived in group 1. The results are 

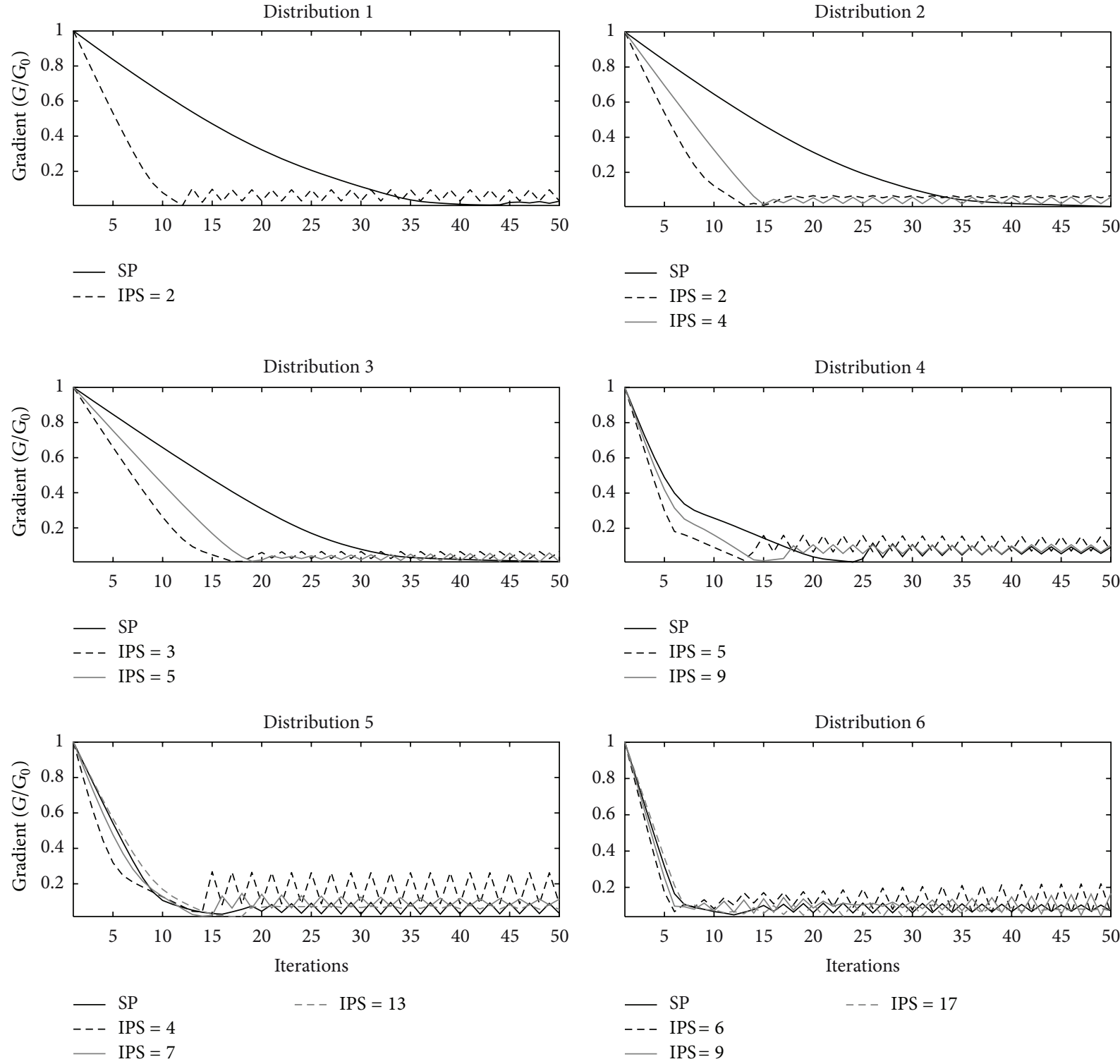

FIGURE 5: The iteration histories of the $L_{2}$ norms of gradients of the cost functions with respect to OBCs for group 1.

shown in Figure 6, which show that the inverted distributions are in good consistency with the prescribed distributions. And the results demonstrate that the 6 kinds of prescribed distributions are all inverted successfully.

The iteration histories of the cost functions and their gradient norms (all values are normalized by their own initial values) are shown in Figure 7 (only the histories of previous 50 iterations are plotted). After 100 iterations, the cost functions and their gradient norms are both reduced by about 1 order of magnitude compared with their initial values. Table 4 shows the MAEs in OBCs before and after assimilation. The MAEs in OBCs are reduced by 1 order of magnitude compared with the values before assimilation. The experimental results indicate that the model can invert the 6 kinds of prescribed distributions of OBCs successfully on a real topography in the Luzon Strait area.

\section{Summary and Conclusions}

The isopycnic-coordinate numerical model for simulation of internal tides with adjoint assimilation method constructed by Chen et al. [17] is developed into a three-layer version. Two groups of ideal experiments are carried out in order to investigate the estimation of spatially varying OBCs.

In group 1, different IPSs are employed to invert 6 kinds of prescribed spatial distributions of OBCs on an ideal topography. After 100 iterations, the cost functions and their gradient norms are reduced by about 2 orders of magnitude and 

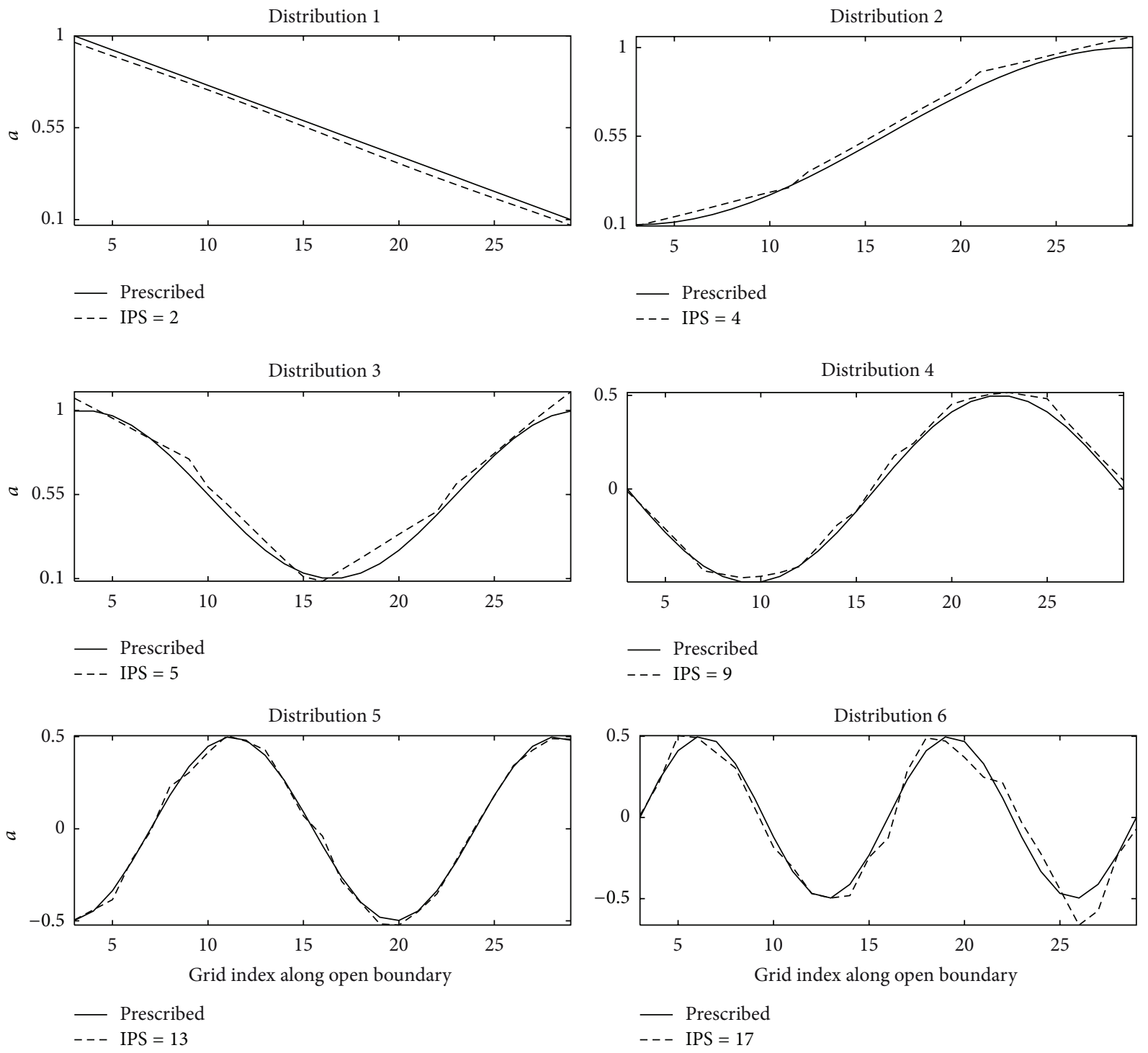

FIGURE 6: Six kinds of prescribed distributions of OBCs (the black solid lines) and their inverted distributions.

by about 1 order of magnitude, respectively, compared with their initial values. Meanwhile, compared with the values before assimilation, the MAEs in OBCs after assimilation are reduced by 1 order of magnitude, and the vector differences in horizontal tidal current after assimilation are reduced by more than $23.53 \%$, respectively. The results of group 1 demonstrate that the inversion test is successful, and this model has a good ability in estimating the spatially varying OBCs. Meanwhile, the use of IPSs can indeed effectively improve the estimation precision of OBCs. Moreover, utilization of fewer independent points is feasible when the distribution of OBCs is simpler, and appropriately more independent points are required when the distribution of OBCs is more complex.

In group 2, the optimal IPSs selected from group 1 are used to invert the 6 kinds of prescribed distributions of OBCs, and the real topography in the Luzon Strait area is installed. The cost functions and their gradient norms after 100 iterations are both reduced by about 1 order of magnitude compared with their initial values. The MAEs in OBCs are reduced by 1 order of magnitude compared with the values before assimilation. The results of group 2 demonstrate that the model also has a good ability in estimating the spatially varying $\mathrm{OBCs}$ on a real topography, which lays a foundation for the coming real experiments.

In conclusion, the work in this paper shows us that the IPSs can improve the estimation of OBCs which play an important role in this model. Thus, the work is significant to the simulation of internal tides in the South China Sea. In addition, some particular choices, such as the nonuniformly distributed independent points and the weighted coefficients of the interpolation, can also be employed by other IPSs in the work of parameter estimation. However, the performance 

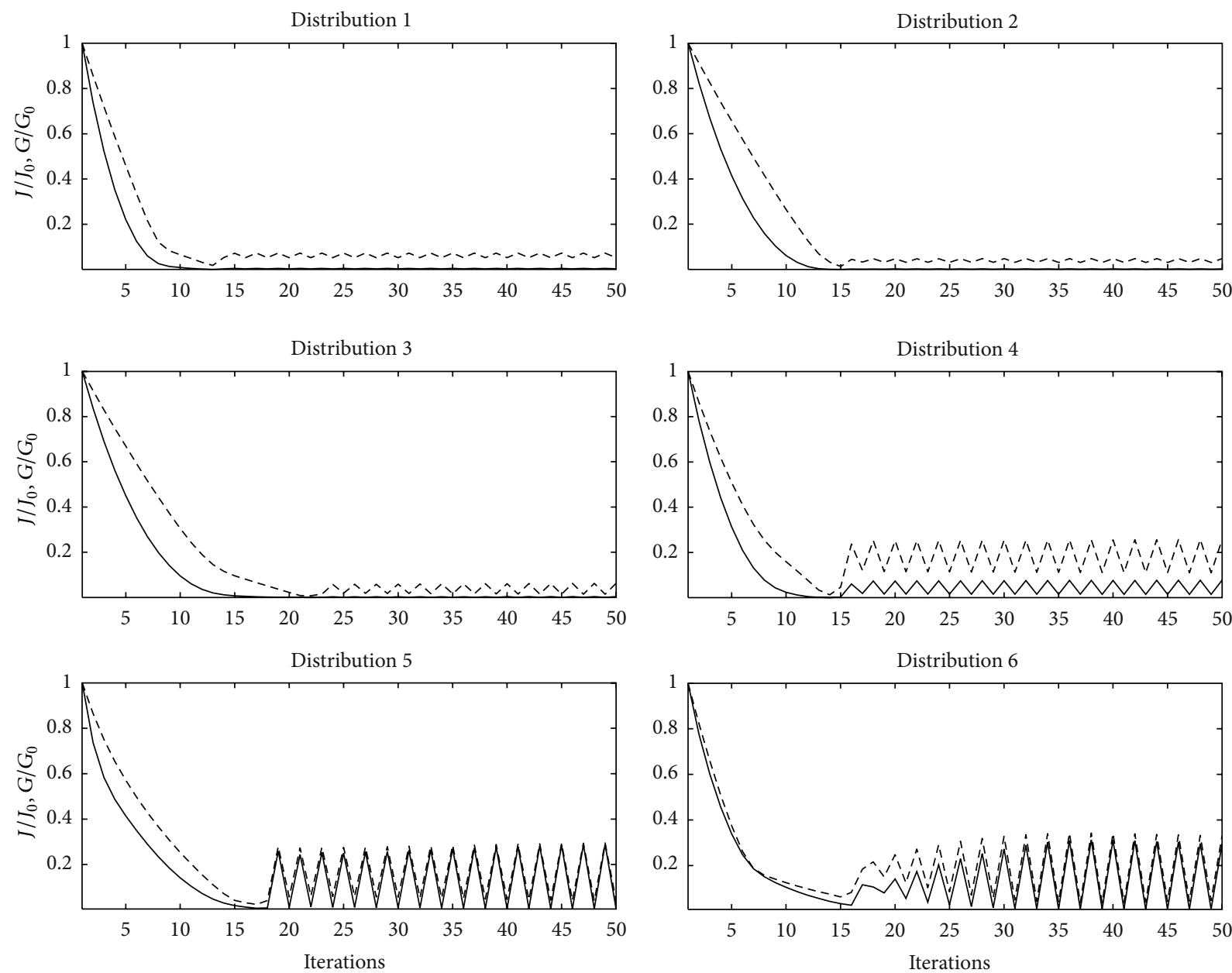

FIGURE 7: The iteration histories of the cost functions and their gradient norms with respect to OBCs for group 2.

TABLE 4: The MAEs in OBCs before assimilation (BA) and after assimilation (AA).

\begin{tabular}{lcccccc}
\hline Distributions & 1 & 2 & 3 & 4 & 5 & 6 \\
\hline BA & 0.550 & 0.550 & 0.567 & 0.305 & 0.326 & 0.305 \\
AA & 0.029 & 0.031 & 0.043 & 0.025 & 0.018 & 0.061 \\
\hline
\end{tabular}

of IPS is likely improved in a practical application, further highlighting its advantage.

\section{Acknowledgments}

The authors acknowledge the support of the Major State Basic Research Development Program of China through Grant 2013CB956500, the National Natural Science Foundation of China through Grant is 41076006, and 41206001, the Natural Science Foundation of Jiangsu Province of China through grant BK2012315 and the Fundamental Research Funds for the Central Universities Grants 201261006 and 201262007.

\section{References}

[1] G. D. Egbert and R. D. Ray, "Significant dissipation of tidal energy in the deep ocean inferred from satellite altimeter data," Nature, vol. 405, no. 6788, pp. 775-778, 2000.

[2] Y. Niwa and T. Hibiya, "Numerical study of the spatial distribution of the M2 internal tide in the Pacific Ocean," Journal of Geophysical Research C, vol. 106, no. 10, pp. 22441-22449, 2001.

[3] G. S. Carter, M. A. Merrifield, J. M. Becker et al., "Energetics of M2 barotropic-to-baroclinic tidal conversion at the Hawaiian Islands," Journal of Physical Oceanography, vol. 38, no. 10, pp. 2205-2223, 2008.

[4] M. H. Alford, J. A. Mackinnon, J. D. Nash et al., "Energy flux and dissipation in luzon strait: two tales of two ridges," 
Journal of Physical Oceanography, vol. 41, no. 11, pp. 2211-2222, 2011.

[5] R. W. Lardner, "Optimal control of open boundary conditions for a numerical tidal model," Computer Methods in Applied Mechanics and Engineering, vol. 102, no. 3, pp. 367-387, 1993.

[6] R. W. Lardner, "Optimal assimilation of current and surface elevation data in a two-dimensional numerical tidal model," Applied Mathematical Modelling, vol. 19, no. 1, pp. 30-38, 1995.

[7] I. Shulman, "Local data assimilation in specification of open boundary conditions," Journal of Atmospheric and Oceanic Technology, vol. 14, no. 6, pp. 1409-1419, 1997.

[8] I. Shulman, J. K. Lewis, A. F. Blumberg, and B. N. Kim, “Optimized boundary conditions and data assimilation with application to the M2 tide in the yellow sea," Journal of Atmospheric and Oceanic Technology, vol. 15, no. 4, pp. 1066-1071, 1998.

[9] A. Zhang, E. Wei, and B. B. Parker, "Optimal estimation of tidal open boundary conditions using predicted tides and adjoint data assimilation technique," Continental Shelf Research, vol. 23, no. 11-13, pp. 1055-1070, 2003.

[10] J. Nycander, A. M. Hogg, and L. M. Frankcombe, "Open boundary conditions for nonlinear channel flow," Ocean Modelling, vol. 24, no. 3-4, pp. 108-121, 2008.

[11] A. W. Heemink, E. E. A. Mouthaan, M. R. T. Roest, E. A. H. Vollebregt, K. B. Robaczewska, and M. Verlaan, "Inverse 3D shallow water flow modelling of the continental shelf," Continental Shelf Research, vol. 22, no. 3, pp. 465-484, 2002.

[12] V. Taillandier, V. Echevin, L. Mortier, and J. Devenon, "Controlling boundary conditions with a four-dimensional variational data-assimilation method in a non-stratified open coastal model," Ocean Dynamics, vol. 54, no. 2, pp. 284-298, 2004.

[13] N. Ayoub, "Estimation of boundary values in a North Atlantic circulation model using an adjoint method," Ocean Modelling, vol. 12, no. 3-4, pp. 319-347, 2006.

[14] I. Y. Gejadze and G. J. M. Copeland, "Open boundary control problem for Navier-stokes equations including a free surface: adjoint sensitivity analysis," Computers and Mathematics with Applications, vol. 52, no. 8-9, pp. 1243-1268, 2006.

[15] J. Zhang and X. Lu, "Inversion of three-dimensional tidal currents in marginal seas by assimilating satellite altimetry," Computer Methods in Applied Mechanics and Engineering, vol. 199, no. 49-52, pp. 3125-3136, 2010.

[16] E. Kazantsev, "Boundary conditions control for a shallow-water model," International Journal for Numerical Methods in Fluids, vol. 68, no. 5, pp. 625-641, 2012.

[17] H. B. Chen, C. B. Miao, and X. Q. Lv, "A three-dimensional numerical internal tidal model involving adjoint method," International Journal for Numerical Methods in Fluids, vol. 69, no. 10, pp. 1584-1613, 2012.

[18] A. Z. Cao, Z. Guo, and X. Q. Lv, "Inversion of two-dimensional tidal open boundary conditions of M2 constituent in the Bohai and Yellow Seas," Chinese Journal Oceanology and Limnology, vol. 30, no. 5, pp. 868-875, 2012.

[19] Z. Guo, A. Z. Cao, and X. Q. Lv, "Inverse estimation of open boundary conditions in the Bohai Sea," Mathematical Problems in Engineering, vol. 2012, Article ID 628061, 9 pages, 2012.

[20] A. Z. Cao, H. B. Chen, J. C. Zhang, and X. Q. Lv, "Optimization of open boundary conditions in a 3D internal tidal model with the adjoint method around Hawaii," Abstract and Applied Analysis, vol. 2013, Article ID 950926, 11 pages, 2013.

[21] H. Yuan and C. H. Wu, "An implicit three-dimensional fully non-hydrostatic model for free-surface flows," International
Journal for Numerical Methods in Fluids, vol. 46, no. 7, pp. 709$733,2004$.

[22] X. Lu and J. Zhang, "Numerical study on spatially varying bottom friction coefficient of a 2D tidal model with adjoint method," Continental Shelf Research, vol. 26, no. 16, pp. 1905-1923, 2006.

[23] J. Zhang and X. Lu, "Parameter estimation for a three-dimensional numerical barotropic tidal model with adjoint method," International Journal for Numerical Methods in Fluids, vol. 57, no. 1, pp. 47-92, 2008.

[24] J. Zhang, X. Lu, P. Wang, and Y. P. Wang, "Study on linear and nonlinear bottom friction parameterizations for regional tidal models using data assimilation," Continental Shelf Research, vol. 31, no. 6, pp. 555-573, 2011. 


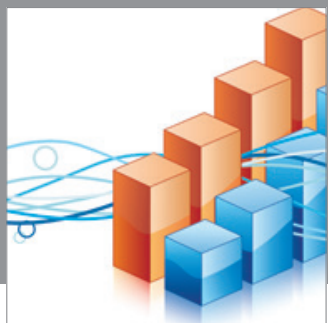

Advances in

Operations Research

mansans

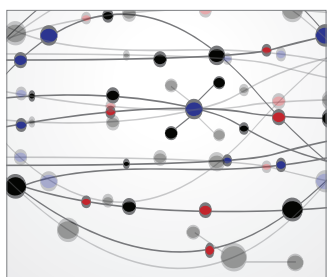

The Scientific World Journal
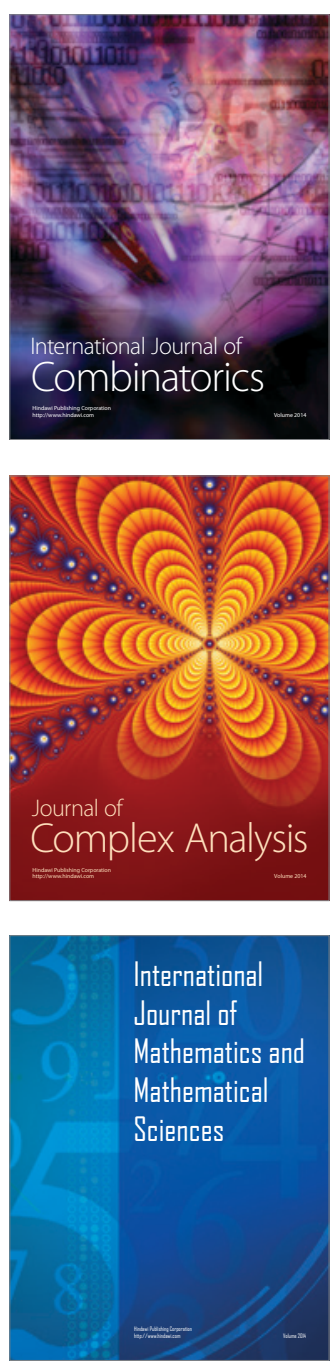
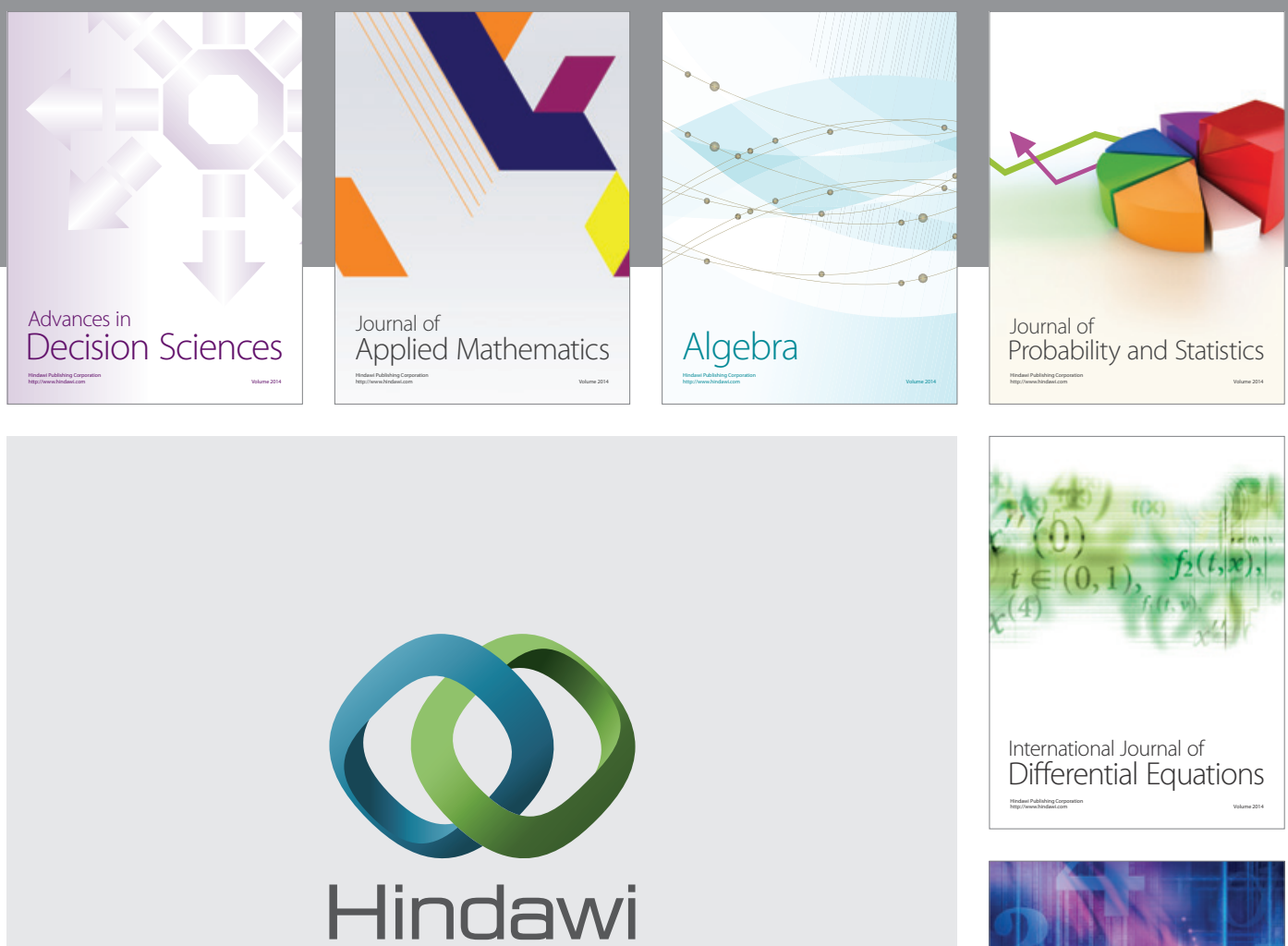

Submit your manuscripts at http://www.hindawi.com
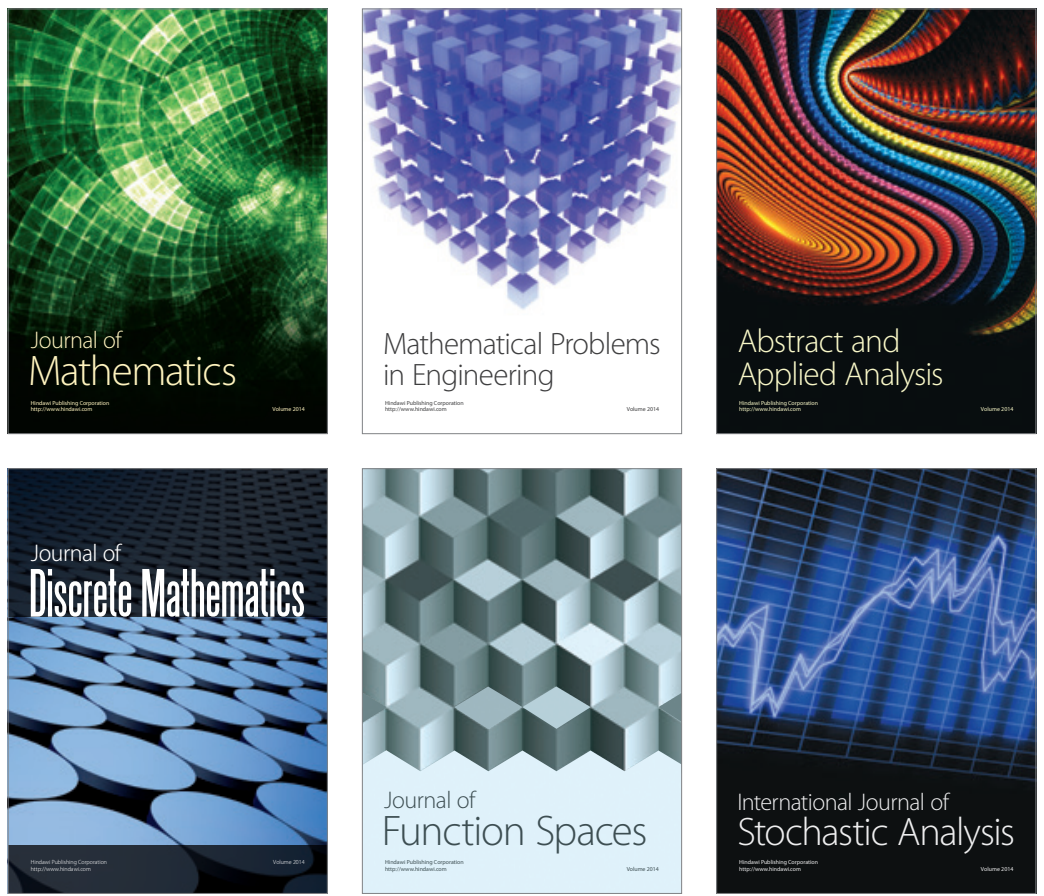

Journal of

Function Spaces

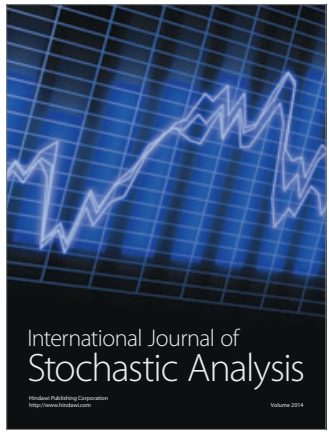

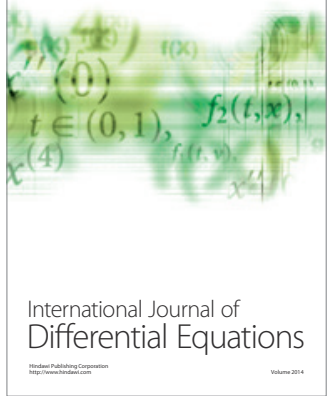
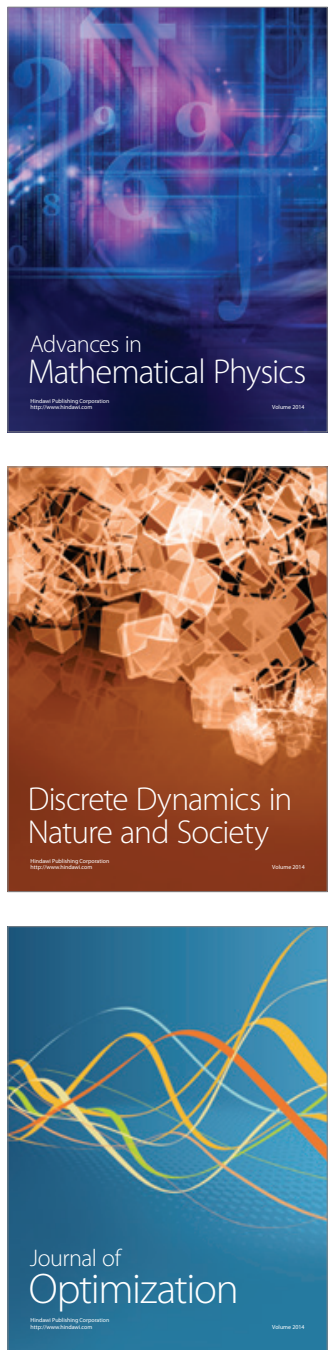